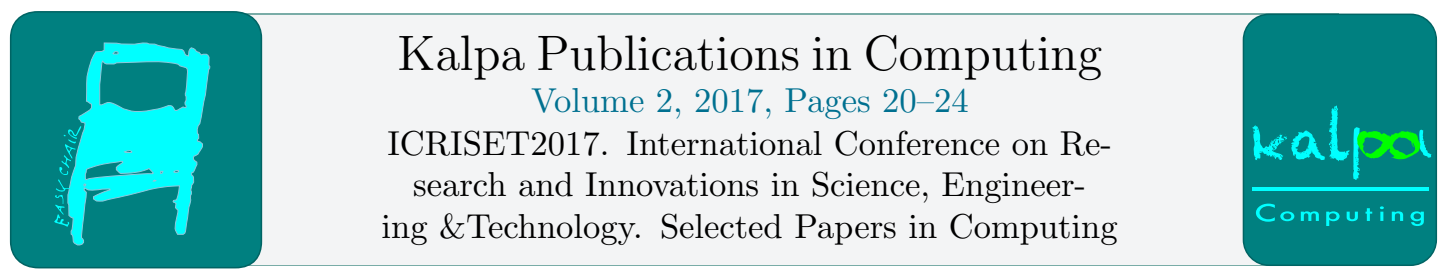

\title{
Revisiting intersection of nine-point circle in the universal mode using novel computing tools (Revisiting Schrödder's findings)
}

\author{
J.K. Chauhan ${ }^{1}$ and R.B. Gandhi ${ }^{2}$ \\ ${ }^{1}$ Department of Electrical Engineering, BVM Engineering College, Vallabh Vidyanagar 388 120, \\ Gujarat, India \\ jkchauhan@bvmengineering.ac.in \\ 2 Department of Mathematics, BVM Engineering College, Vallabh Vidyanagar 388 120, Gujarat, \\ India \\ rbgandhi@bvmengineering.ac.in
}

\begin{abstract}
The geometries of points, lines, line segments, triangles and circles have been studied at depth by many learned mathematicians, scholars and researchers for a long period of time. Among these geometries the geometrical properties of nine-point circle is indeed interesting, fascinating and glamorous also. The curiosity present in human mind have motivated the aforesaid community to explore the properties of more than one nine-point circle emerging from four arbitrarily chosen points. In this paper the authors have attempted to simulate a few of them in a user friendly environment and bringing in light a case which is yet not either studied or not found in the prevailing literature, as regards Schrödder's findings.

Key words: Nine-point circle, ortho-centric points, collinear points, MATLAB
\end{abstract}

\section{Introduction}

The NPC in the world of geometry is like a moon in the sky. This is because of its interesting, fascinating and much more than that complex as regards the proof of its properties. The construction of an NPC has been carried out by numerous learned mathematicians, researchers and scholars. By the curiosity of the afore-said communities of researchers more extensive studies have also come into picture. This includes the emergence of the four NPC coming into the picture on selection of four arbitrarily chosen points and their findings are stated as follow: The four arbitrarily chosen points describe four basic reference triangles. Their NPCs are always concurrent in a point. Further, if the four arbitrarily chosen points are ortho-centric then the four basic triangles have an NPC common to all. The above statements though stated, discussed and proved as well, they are found to be still reserved in its universality. Before this universality is brought in light, the following section provides the selection of geometrical tools and software. Selection of co-ordinate system and software The Cartesian co-ordinate system is substantially old and well tested to verify and prove very interesting facts of geometry for a long period of time. This is because of the simplicity of the system and availability of a large number of results

R. Buyya, R. Ranjan, S. Dutta Roy, M. Raval, M. Zaveri, H. Patel, A. Ganatra, D.G. Thakore, T.A. Desai, Z.H. Shah, N.M. Patel, M.E. Shimpi, R.B. Gandhi, J.M. Rathod, B.C. Goradiya, M.S. Holia and D.K. Patel (eds.), ICRISET2017 (Kalpa Publications in Computing, vol. 2), pp. 20-24 
as regards the geometry problems. Because of this here also it is selected wisely to formulate the solution of the problem in the simulation environment. Similarly, MATLAB is one of the most popular and user-friendly software to address the problems of various fields of science and technology. It has also a strong library of commands to draw geometric figures simply using simple syntax. Therefore MATLAB is used here also to obtain required simulations. Such simulations have been prepared in this environment, using the MATLAB commands by numerous learned researchers and scholars to solve the complex geometry problems in engineering and mathematics [[4], [9], [13]].

\section{Simulations}

Simulation steps for case I and case II, have been listed as follows:

\section{Case I}

1. Generate a matrix $R(4,2)$, using random number generator command in MATLAB.

2. Scale the matrix, using a suitable multiplier.

3. Name the elements $R(i, 1)$ as the $x_{i}$ and $R(i .2)$ as the $y_{i}$ co-ordinates for $i=1,2,3,4$ of the four arbitrarily selected points.

4. Define the four basic reference triangles and construct their NPCs using the NPC function.

5. Test the concurrence of the NPCs constructed in step 4 and verify the result.

\section{Case II}

1. Generate a matrix $R(3,2)$, using random number generator command in MATLAB.

2. Scale the matrix, using a suitable multiplier.

3. Name the elements $R(i, 1)$ as the $x_{i}$ and $R(i .2)$ as the $y_{i}$ co-ordinates for $i=1,2,3$ of the three arbitrarily selected points.

4. Obtain the ortho-centre of the triangle described by the above three points.

5. The above three points and the ortho-centre as fourth point, collectively describes an ortho-centric system of four-points.

6. Define the four basic reference triangles and construct their NPCs using the NPC function.

7. Test the concurrence of the NPCs constructed in step 4 and verify the result.

\section{Case III}

The previous section describes the selection of the four arbitrary points and the simulations confirm to the results obtained earlier. But the user has still a freedom to choose three points collinear out of the four. In this case, there can exists only three basic reference triangles and thus three basic NPCs come into picture. But very interestingly they are also concurrent at a point (namely, at the foot of the common altitude among the three basic triangles). This case is not much found in the literature pertaining to this.

Simulation of this case can be carried out through the following simulation steps:

1. Generate a matrix $R(3,1)$, using random number generator command in MATLAB. 
2. Generate a random number $c$, using the random number generator.

3. Scale the matrix $R(3,1)$, using a suitable multiplier.

4. Scale the constant $c$ also using a suitable multiplier.

5. Name the elements $R(i, 1)$ as the $x_{i}$ for $i=1,2,3$ of the three arbitrarily selected points.

6. Obtain the $y_{i}$-coordinates corresponding to $x_{i}$ using the line equation $y=m x+c$.

7. Generate the fourth point, using the method described above (This is the users choice).

8. Define the three basic triangles and construct their NPCs using NPC function.

9. Test the concurrence of the above three NPCs constructed in step 8 and verify the result.

\section{Case IV}

In this case all four points are linear. Therefore no scope of discussion as no triangle formation will occur.

The above discussion in the form of the corresponding diagrams is shown in Appendix I.

\begin{tabular}{lllll}
\hline Sr No & $\begin{array}{l}\text { Nature arbitrarily } \\
\text { chosen four } \\
\text { points }\end{array}$ & $\begin{array}{l}\text { Number of } \\
\text { NPC } \\
\text { existing }\end{array}$ & Concurrence & $\begin{array}{l}\text { Number of } \\
\text { concurrent } \\
\text { points }\end{array}$ \\
\hline 1 & $\begin{array}{l}\text { Four coplanar points } \\
\text { where no three } \\
\text { are collinear }\end{array}$ & Four & $\begin{array}{l}\text { They are concurrent } \\
\text { at a point }\end{array}$ & One \\
\hline $\begin{array}{l}\text { Four coplanar points } \\
\text { where three are } \\
\text { collinear }\end{array}$ & Three & $\begin{array}{l}\text { They are concurrent } \\
\text { at a point }\end{array}$ & One \\
\hline $\begin{array}{l}\text { Four coplanar ortho- } \\
\text { centric points } \\
\text { All the four points } \\
\text { are collinear }\end{array}$ & Four & None & $\begin{array}{l}\text { All the NPCs are } \\
\text { common } \\
\text { No scope of } \\
\text { discussion }\end{array}$ & $\begin{array}{l}\text { Infinitely } \\
\text { many }\end{array}$ \\
& & $\begin{array}{l}\text { No scope of } \\
\text { discussion }\end{array}$ \\
\hline
\end{tabular}

Table 1:

\section{Future Scope}

As stated in case IV, if the four collinear points are chosen so as to match the inner division ratio as observed in the discussion of Euler line, what about the existence of an NPC?

\section{Acknowledgments}

The authors are thankful to BVM Engineering College and CVM for infrastructure support. The authors are also thankful to the organizing team of ICRISET-2017 for providing the opportunity to present this work. The authors are also thankful to the faculty members of mother departments and friends for the fruitful discussion. Last but not the least, a sincere thanks to the family members for their patience and tolerance. 


\section{References}

[1] Aiyar V.R. A general theorem on the Nine-point circle. Proceedings of the Edinburgh Mathematical Society, Volume 15, 74 - 75, 1896. DOI: https://doi.org/10.1017/S0013091500032016

[2] Baker H.F. Principles of Geometry, Volume II, Cambridge, Cambridge University Press, 1922.

[3] Boyer C.B. A History of Mathematics, New York: John Wiley \& Sons, 1968.

[4] Chauhan J.K. and Gandhi R.B. Simulation of Nine-Point Circle using novel computing tools and investigating the associated useful properties. Inroads, Volume 5, No. 1, 305 - 309, 2016.

[5] Coxeter H.S.M. and Greitzer S.L. Geometry Revisited. Washington, DC: The Mathematical Association of America, 1967.

[6] De Villiers M.D. A generalization of the nine point circle and the Euler line. Pythagoras, 62, 31 35, 2005.

[7] De Villiers M.D. The nine-point ellipse: a rediscovery and proof by computer. International Journal of Mathematical Education in Science and Technology, Vol. 37, No. 1, 7 - 14, 2006.

[8] Eves H. (Ed.) Mathematical Circles. Boston: Prindle, Weber \& Schmidt, 1969.

[9] Serb G.P. and Serb I.P. Numerical Approach of the Partial Magnetic Characteristics method used to find the voltage regulation in the design algorithm of a synchronous generator. 13th International Conference on Optimization of Electrical and Electronic Equipment (OPTIM), ISBN-978-1-46731653-8/12-IEEE, 745 - 750, 2012.DOI: 10.1109/OPTIM.2012.6231914

[10] Jingcheng Tong and Sidney Kung. Twelve new points on the nine point circle. Published by the applied probability trust, 1996.

[11] Kimberling C. Triangle Centers and Central Triangles. Congr. Numer. 129, 1 - 295, 1998.

[12] Mackay J.S. History of the Nine-Point Circle. Proc. Edinburgh Math. Soc. 11, 19 - 61, 1892.

[13] Patel D.K., Chauhan J.K., Patel A.R. and Mishra N.G. A user friendly simulation for transformer vector group. IEEE International Conference, CSNT-2012, 2012. DOI: 10.1109/CSNT.2012.178

[14] Patel D.K., Chauhan J.K., Patel A.R. and Mishra N.G. Revisiting ZPF method using novel computing tools. International Journal on Recent and Innovation Trends in Computing and Communication, Volume 4, Issue 4, 787 - 791, 2016.

[15] Schröder E. M. Zwei 8-Kreise-Sätze für Vierecke.” Mitt. Math. Ges. Hamburg 18, 105 - 117, 1999.

[16] www.mathworks.com 


\section{Appendix I}

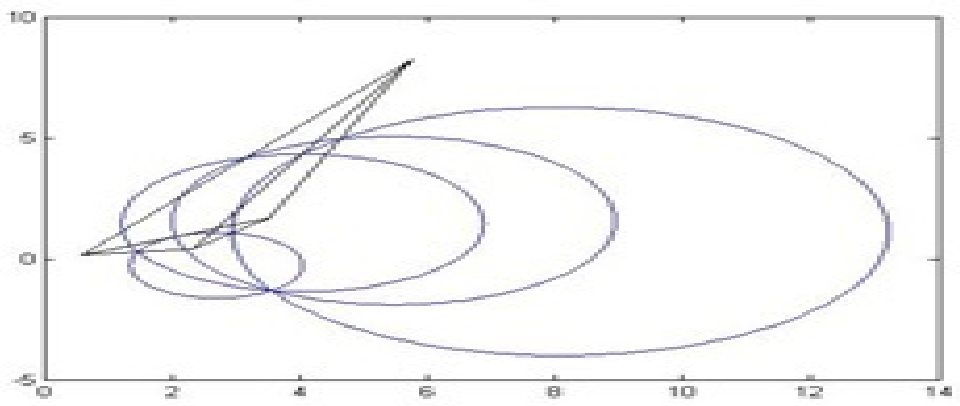

Figure 1: Four coplanar points where no three are collinear

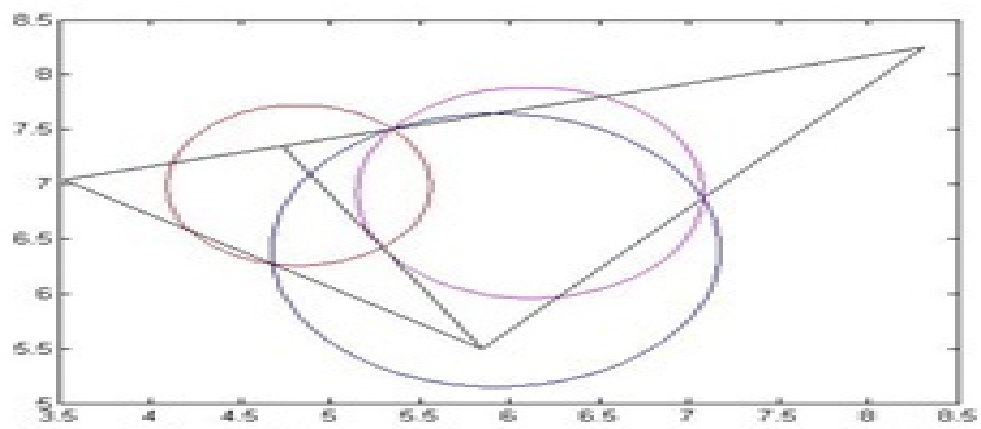

Figure 2: Four coplanar points where three are collinear

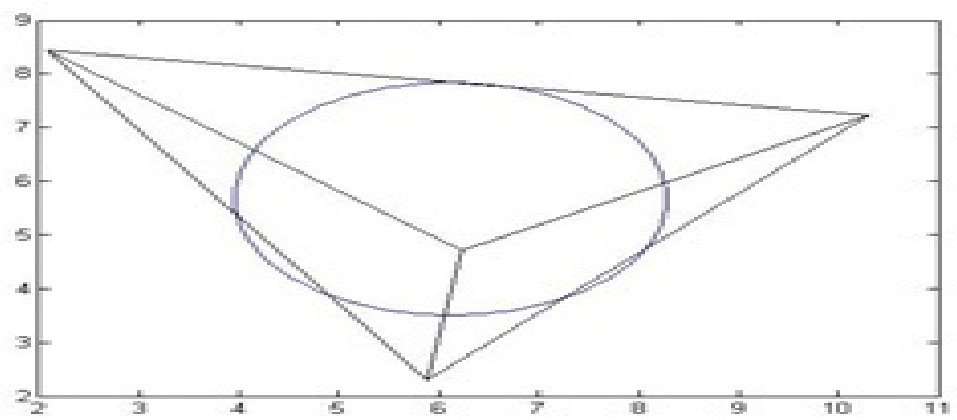

Figure 3: Four coplanar ortho-centric points 